Neurosurg Focus 8 (2):Introduction, Click here to return to Table of Contents

\author{
Introduction to Neurosurgical Focus Volume 8, Issue 2, February 2000 \\ Topic Editor: Richard S. Polin, M.D. \\ Positron Emission Tomography Scanning in Clinical Medicine
}

\title{
Applications of positron emission tomography in neurosurgery: past, present, and future
}

\author{
Richard S. Polin, M.D., David L. Lilien, M.D., Jose Menendez, M.D., \\ AND ANIL NANDA, M.D. \\ Departments of Neurosurgery and Radiology, and Biomedical Research Foundation of Northwest \\ Louisiana, Louisiana State University Health Sciences Center, Shreveport, Louisiana
}

\begin{abstract}
Any novel technological innovation is dependent more on the ingenuity of its users than its inherent properties and potential flaws. Positron emission tomography (PET) is unique in this regard because the limitations of this modality are defined by the ability of its users to identify tracers that will aid in the diagnosis of intracerebral processes. The limitations of PET scanning lay in the extent of tissue resolution and in the currently relatively small number centers equipped to perform PET scanning.

In this issue of Neurosurgical Focus, various applications of PET scanning are detailed. The traditional use of this technology in neurosurgery has been to determine the metabolic nature of cerebral neoplastic lesions so as to help differentiate neoplastic from benign or infectious processes. This determination, however, has not been foolproof, and the technique has been further refined to maximize diagnostic yield.

Nonetheless, the utility of PET scanning continues to grow. New applications have allowed for the precise measurements of cerebral blood flow, helping the neuroscientist understand the functional organization of cortex, the pathophysiology of normal-pressure hydrocephalus, and changes in cerebral blood flow following traumatic injury. Other radiotracers such as fluorodopa allow assessment of the metabolic state of cerebral tissue transplants in restorative neurosurgical procedures.

In this introduction, all of these issues will be considered, and a historical perspective and the potential future uses of this technology will be provided. Technology moves so quickly that new instruments are routinely introduced. The authors will try to assess what properties, in the 25-year history of PET scanning, will need to be improved to keep it as a cutting-edge technology and expand its clinical role.
\end{abstract}

\section{HISTORICAL PERSPECTIVE}

Currently, the most widely available method of brain imaging in which radionuclides are used, involves mapping the distribution of intravenously injected radiotracer by acquiring images in a full $360^{\circ}$ around the patient's head. With this technique, called single-photon emission tomography scanning, the tracers usually consist of a molecule with a defined biological behavior labeled with a radioactive element that emits a single gamma ray per nuclear disintegration. Examples include the flow tracers ECD or HMPAO labeled with technetium-99m in which uptake of the lipid-soluble tracer is proportional to blood

$\mathrm{CBF}=$ cerebral blood flow; $\mathrm{CT}=$ computerized tomography; FDG = fluorodeoxyglucose; $\mathrm{MR}=$ magnetic resonance; $\mathrm{PET}=$ positron emission tomography; flow, or the ionic tracer thallium-201 that concentrates, to some degree, in tumors and is excluded from normal brain tissue. Biologically inert, highly lipid-soluble tracers such as ${ }^{133} \mathrm{Xe}$ gas can be used to measure $\mathrm{CBF}$, with somewhat specialized equipment to measure the washout rate of the tracer after inhalation. One advantage of these so-called single-photon-emitting tracers is that they have halflives adequate for distribution and storage at the site of injection and can be visualized, in most cases, using conventional nuclear imaging equipment, which is present in essentially all acute-care facilities. One disadvantage is that their versatility is limited; instead of actually measuring a biological process directly, the behavior of the tracer, which is proportional to the biological parameter one would prefer to study directly, is being observed. Furthermore, they are not quantitative except in the case of ${ }^{133} \mathrm{Xe}$ CBF studies. 
In PET scanning tracers are used that have very short halflives ( $\leq 2$ hours) produced by cyclotrons. In addition to the requirement for an on-site or nearby cyclotron for isotope production, the scanners used to obtain the PET images are more complex and more expensive than standard nuclear imaging devices. Nonetheless, in comparison with single-photon emission studies, PET has major advantages related both to the types of radiotracers used and to the intrinsic properties of the scanning devices. ${ }^{9}$

Positron-emitting isotopes are produced by proton or deuteron bombardment of a stable target element (for example oxygen-18, a naturally occurring form of the element oxygen) in a cyclotron, producing an unstable element (fluorine-18 in the case of bombardment of oxygen-18), which can only become stable by emitting charge and mass in the form of a positron from its nucleus. Positrons are positively charged electrons (antimatter) that move a very short distance from the site of emission and interact with one of the myriad electrons present in tissues. This interaction results in annihilation of the matter (electron) and antimatter (positron), whereby the mass of two particles is converted into energy which, in the form of two gamma rays of 511-kiloelectron volts energy, travels away from the site of the annihilation at essentially $180^{\circ}$ angles from each other. These gamma-ray pairs "in coincidence" are detected by a ring of detectors by using timing circuitry that projects the location of the event producing them along a line between the two detector elements. ${ }^{23}$ Many millions of such events are used to produce cross-sectional images in much the same way that parallel beams of X-rays are used to produce CT scans. Positron emission tomography devices are much more efficient than single-photon devices, and furthermore, the tracer distribution can be readily quantitated. By applying knowledge of the physiological and biochemical features of the tracer, metabolic reactions, receptor ligand densities, or other physiological processes can be very accurately measured.

The use of positron tracers in biology actually predates the use of beta-emitting tracers. ${ }^{20}$ Carbon- 11 was used, for example, in pioneering and Nobel Prize-winning research conducted by Calvin and Kamen in the 1930s and 1940s to elucidate the mechanism of photosynthesis. The first use of carbon-11 in humans dates back to the 1940s. It was not until the development in the 1960s of imaging devices capable of mapping radiotracer distribution in living patients that these neuroimaging studies were performed in medical research in patients and volunteers. Positron emission tomography scanning as we now know it was developed by TerPogossian, Phelps, and their colleagues in the mid-1970s. ${ }^{13,21}$ Unlike MR imaging which was developed around the same time, PET remained essentially a research tool until the late $1980 \mathrm{~s} .{ }^{14}$ Since that time, there has been steady development of both research and clinical uses of this technology. The current difficult regulatory- and reimbursement-related climate that surrounds diagnostic imaging has slowed the development of clinical applications of PET technology, but the influx of new radiotracers will continue to augment the utility of this technology.

\section{Use of Tracers}

Positron-emitting tracers of biological interest include oxygen-15 (halflife 120 seconds), nitrogen-13 (halflife 10 minutes), and fluorine-18 (halflife 120 minutes). Oxygen15 can be used as a flow tracer in the form of oxygen- 15 water, as a tracer of oxidative metabolism in the form of molecular oxygen, or as a tracer of blood volume in the form of carbon monoxide that binds to the hemoglobin of red blood cells. The elegance of this tracer in mapping and quantitating these biological parameters has made it an extremely useful tracer in research experiments. Much of what is currently known about the mapping of cerebral function was acquired using this tracer. The very short halflife and complex handling requirements have limited the use of oxygen-15 to a small number of centers. Whereas nitrogen-13 in the form of ammonia gas is used as a flow tracer in the heart, it is not used as such in neurological studies. In the form of ${ }^{13} \mathrm{~N}$-labeled amino acids, it can be used as a tracer of protein synthesis, and it is used, to some extent, in neurooncology studies. Carbon11 is an extremely versatile tracer that has been synthesized into hundreds of compounds, including receptor ligands (including neurotransmitters), hormones, drugs, and hundreds of different metabolic precursors. The only limitations have been the imagination and ingenuity of the chemists developing the rapid syntheses needed for in vivo use. Nonetheless, the short halflife limits its use to facilities with cyclotrons.

Fluorine-18 is the most widely used positron tracer. Although it is not itself an important biological element, by relatively simple and rapid synthetic methods fluorine can be substituted for hydrogen or hydroxyl groups in a wide variety of compounds. It has a longer halflife than other tracers used in clinical PET studies and can be shipped to sites at which scans are being obtained without cyclotrons, usually within a transportation radius of 2 hours. Fluorine-18 also has excellent imaging characteristics related to its relatively short positron range in tissue, allowing images to be of higher resolution than those obtained using the aforementioned other single photon or positron tracers. It is most commonly used in the form of FDG. This application evolved from the Nobel Prize-winning work of Sokoloff and colleagues ${ }^{17}$ at the National Institues of Health. Fluorodeoxyglucose is a glucose ana$\log$ and, as such, is transported into cells by endogenous glucose transporters. Once it becomes intracellular, it is phosphorylated by hexokinase but undergoes no further metabolic change. Hence, it is trapped within cells, and this allows scanning to be performed at a convenient time (usually 1 hour) after injection. Because neural cells can only use glucose as their energy substrate, FDG is a very useful tracer of overall neural function. Because an increase in glucose metabolic rate accompanies all neural function, FDG is also a useful tracer for the mapping of a site at which it has been injected while a function is elicited and continues throughout the period during which a high concentration of FDG circulates (usually 20 minutes). This phenomenon also allows the use of FDG in cases of epilepsy in which ictal injections demonstrate increased FDG uptake and interictal injection demonstrates decreased uptake. In addition, the noted biochemist Warburg ${ }^{22}$ demonstrated in the late 1920s that an increase in glucose levels was seen in neoplastic tissue as in contrast with the normal tissue from which it derived. This seminal observation has led to the use of FDG in brain 
tumors and, with the development of imagers capable of visualizing the entire body, has led to an explosion in its application in oncology over the past several years. A number of emerging applications involve the use of FDG as an index of the density of neural cells in such processes as Alzheimer's disease.

Other tracers incorporating fluorine-18 are now in use and others are being developed. Fluorodopa allows imaging of the striatum, and a variety of neuroligands, amino acids, drugs, and cell-proliferation tracers incorporating this isotope are beginning to be applied clinically. Although FDG is, at this time, the most widely used radiotracer in patients undergoing neurosurgical procedures, the clinical role of these other compounds will undoubtedly expand.

\section{SYNOPSIS OF PAPERS IN THIS ISSUE OF NEUROSURGICAL FOCUS}

In this edition of Neurosurgical Focus, we are fortunate to be able to present a diverse collection of applications of PET in neurosurgery. The reviewers, whose experience encompasses clinical neurosurgery, neurooncology, and PET neuroimaging, have done an outstanding job selecting a diverse assortment of papers. Uses of PET in these papers fall into two categories: diagnostic imaging and functional testing.

The use of PET to visualize cerebral lesions has focused on differentiating neoplastic hypermetabolic processes from nonneoplastic entities such as radiation-induced necrosis. As more experience is gained and new tracers are developed, the ability to predict more accurately the behavior of the lesion will be augmented. Massager and colleagues from Erasme Hospital in Belgium provide a considerable series of patients in whom PET and MR imaging were used to determine the best site at which to obtain a stereotactic biopsy. Although the diagnostic predictive ability of PET and MR imaging either together or combined was limited, the authors used areas of high FDG uptake on PET to target the best location at which to acquire the sample, obviating the need for multiple passes. Menendez and associates demonstrate that PET scanning can also aid in the differential diagnosis of solitary mass lesions in patients with acquired immune deficiency syndrome. The ability to differentiate the most common pathological entities, toxoplasmosis and lymphoma, may reduce the need for conducting biopsy procedures in these patients. Matheja and colleagues remind us that no imaging modality is foolproof by describing a patient who, 20 years after undergoing tumor resection and radiotherapy, experienced frontal lobe hypermetablolism, which was determined histopathologically to represent radiation necrosis.

The ability of PET scanning to provide functional localization is also highlighted in this series of papers. Kaplan and associates report a small series of pediatric patients with benign tumors who underwent language and speech testing by using $\left[{ }^{15} \mathrm{O}\right] \mathrm{H}_{2}$ uptake prior to tumor resection. Their ability to combine MR and PET data into a single image allowed precise stereotactically determined resections to be performed, leaving eloquent cortex undisturbed. The authors conclude that the disadvantages of PET, as compared with functional MR imaging, of poorer resolution and radiation exposure are counterbalanced by two advantages: less movement artifact and the ability to grade tumors. Of course, functional localization is only helpful if the results correspond to intraoperative cortical mapping. Bittar and colleagues elegantly performed $\left[{ }^{15} \mathrm{O}\right]$ $\mathrm{H}_{2}$ uptake mapping of somatosensory cortex and then correlated pre-operative localization with intraoperative findings during craniotomy in awake patients. Despite the limits of spatial resolution of PET and the potential errors of frameless stereotaxy, areas of significant PET-activated foci correlated with cortical stimulation sites in 22 of 23 cases. They describe thresholds of PET stimulation that may eliminate the need for awake craniotomies.

The use of PET to assess cortical activation may also be useful in functional neurosurgery. Pre- and postoperative imaging of $\left[{ }^{15} \mathrm{O}\right] \mathrm{H}_{2}$ uptake has been used to define the effects of functional surgery in cases of Parkinson's disease, ${ }^{4}$ obsessive compulsive disorder, and pain syndromes. Davis and associates report on the changes on pre- and postoperative PET scanning in patients undergoing placement of thalamic stimulators for the treatment chronic pain syndromes. The investigators found that deep brain stimulators caused activation of the contralateral anterior cingulate cortex, a known center involved in pain and analgesia. Klinge and colleagues use $\left[{ }^{15} \mathrm{O}\right] \mathrm{H}_{2}$ uptake to demonstrate that an early improvement in cerebrovascular reserve suggested a favorable prognosis after shunt placement for normal-pressure hydrocephalus.

\section{OTHER TOPICS IN NEUROSURGERY}

\section{Application of PET in Epilepsy}

The use of PET scanning has helped to demonstrate hypometabolism in areas of mesial temporal sclerosis causing epilepsy. Localization of epileptic foci by using $\left[{ }^{18} \mathrm{~F}\right]-$ FDG PET has been used to reduce or eliminate the need for depth electrode monitoring at some centers. ${ }^{3,8}$ Furthermore, functional testing may be performed at the same sitting. Changes revealed on PET scanning have been more impressive than those observed on conventional neuroimaging. Xiong, et al., ${ }^{23}$ have postulated that metabolic changes seen on PET scans may simply precede structural alterations detected on MR imaging. Other tracers such as $\left[{ }^{11} \mathrm{C}\right]-$ flumazenil have been introduced to localize foci more precisely. ${ }^{16}$

\section{Application of PET in Brain Injury}

Positron emission tomography scanning offers theoretical and real assistance in the management of brain injuries. When considering the utility of PET scanning, one must consider the tracer alternatives that are available as well as the complementary data they provide. After traumatic brain injury, there are presumably areas of absolute injury surrounded by areas in a state of potentially reversible ischemia. The source of this reversible ischemia has been ascribed to either vasomotor dysfunction or small vessel vasospasm. Tracers that measure CBF (hydrogen-2 oxygen-15) may provide quantitative information as to the state of these regions as an alternative to xeCT scaning.

Also of concern after brain injuries is regional glucose utilization. In animal models hyperglycolysis is associated 
with activation of excitotoxic amino acid release. Bergsneider and colleagues ${ }^{2}$ have examined hyperglycolysis in humans after brain injury by using $\left[{ }^{18}-\mathrm{F}\right]-\mathrm{FDG}$ PET. They included patients with Glasgow Coma Scale scores of 12 or lower who were 16 years of age and older. Twentyeight patients were evaluated, six of whom also underwent ${ }^{133} \mathrm{Xe}-\mathrm{CBF}$ studies. Global hyperglycolysis was demonstrated in six patients whereas the presence of regional increased glucose was revealed in five.

\section{History of PET in Neurooncology}

Positron emission tomography scanning has been used in the evaluation of brain tumors for over 15 years. The use of FDG PET scanning allows an accurate estimation of the metabolic activity of normal brain function and tumors, based on the different uptakes of labeled deoxyglucose into the cell.

Preoperatively, PET can be used for the grading of tumors, and, postoperatively, for follow up and diagnosis of tumor recurrence. In studies conducted by Di Chiro and associates $^{5}$ they have demonstrated that FDG PET can determine the degree of malignancy by the quantity of glucose uptake. Differentiated cells usually have a lower level of glucose concentration than those found in poorly differentiated tumors.

Patronas, et al., ${ }^{11}$ and Alavi, et al., ${ }^{1}$ have clearly shown the value of FDG PET in predicting the prognosis of patients with brain tumors. In the former study the authors used PET to study 45 patients with high-grade astrocytomas and discovered that a mean survival time of 5 months was shown in patients with higher glucose uptake as compared with 19 months for the patients with lower levels of glucose uptake. Alavi, et al., found that of 29 patients with brain tumors, in those with hypermetabolic tumors the neoplasm tended to behave in a more malignant fashion; in these patients mean survival was 7 months as compared with 33 months for those with hypometabolic lesions.

Prior to the advent of PET scanning, neurological examination and neuroimaging studies were unable to differentiate between both entities, and a surgical biopsy procedure or resection was the only reliable diagnostic tool. The information provided by $\mathrm{CT}$ and MR imaging is based on structural changes and extravasation of contrast media caused by disruption of the blood-brain barrier, which occurs in tumors as well as in radiation-induced necrosis resulting in contrast enhancement. Doyle and colleagues $^{7}$ and Di Chiro and associates ${ }^{6}$ have demonstrated a good correlation between findings on PET and those on pathological examination in tumor recurrence and radiation-induced necrosis. Necrotic tissue appeared as hypometabolic in the abnormal areas noted on MR imaging or CT scanning, whereas tumor recurrence was hypermetabolic.

For the monitoring of response to multimodal treatment, PET can provide information that is otherwise unobtainable. Ogawa, et al., ${ }^{10}$ have demonstrated that the FDG uptake in the tumor decreased an average of $41.3 \%$ when compared with baseline in 80 patients with glioma who underwent 1 month of adjuvant therapy.

\section{New Topics in PET in Neurooncology}

In novel applications of PET scanning, investigators have attempted to tailor treatment of CNS neoplasms based on their metabolism. Spence and colleagues ${ }^{18}$ have described the use of 2-[ $\left.{ }^{11} \mathrm{C}\right]$-thymidine-labeled tracer in CNS gliomas. Thymidine incorporation is known to occur in proliferating CNS neoplasms. The authors found increased $2-\left[{ }^{11} \mathrm{C}\right]$-thymidine uptake in gliomas, including one low-grade lesion that progressed to an aggressive glial neoplasm. Stelzer and associates ${ }^{19}$ found that the volume of abnormal FDG uptake demonstrated on PET scans predicted time to tumor progression. They used areas of gadolinium enhancement and hypermetabolism on FDG PET to guide radiation therapy. Other tracers have been used to detect hypoxia in tumors. Hypoxia makes many tumors relatively resistant to the effects of radiotherapy. Rasey, et al., ${ }^{15}$ have used $\left[{ }^{18} \mathrm{~F}\right]$-fluoromisonidazole as a marker for hypoxia in systemic tumors in a model that may be applicable to intracranial neoplasms. Pirotte, et al., ${ }^{12}$ have discussed using image fusion to allow PET targeting of supratentorial lesions for stereotactic biopsy procedures.

\section{Future Trends}

Although it remains impossible to predict the longevity of any diagnostic method in medicine, there are several features of PET scanning that need to be improved to drive clinical interest.

Availability of Scanners and Tracers. The technology is not universally applicable because of the currently limited number of centers equipped to perform these studies. Many centers are limited by the capabilities of their cyclotrons or chemists and are not able to work with all the available tracers discussed in this paper. For the neurosurgeon, the ability to use hydrogen- 2 oxygen- 15 or a comparable tracer to perform blood flow and functional localization studies is essential to take full advantage of the technology.

Spatial Resolution. As the resolution of the scanners improve, the ability to use PET scanning to target lesions stereotactically or plan radiosurgery will be greatly enhanced.

Image Fusion. The ability to fuse PET scans with CT and MR images is becoming more universally available and will assist the neurosurgeon in understanding the neuroanatomical correlates of PET data.

The novel applications explored by the authors of papers in this edition of Neurosurgical Focus have used advances in resolution and image fusion to augment the limits of the technology. Hopefully, new imaging protocols and tracers will continue to expand these borders and provide new insight into neurosurgical disease processes.

\section{References}

1. Alavi JB, Alavi A, Chawluk J, et al: Positron emission tomography in patients with glioma. A predictor of prognosis. Cancer 62:1074-1078, 1988

2. Bergsneider M, Hovda DA, Shalmon E, et al: Cerebral hyperglycolysis following severe traumatic brain injury in humans: a positron emission tomography study. J Neurosurg 86: 241-251, 1997

3. Chee MW, Morris HH III, Antar MA, et al: Presurgical evaluation of temporal lobe epilepsy using intraictal temporal spikes and positron emission tomography. Arch Neurol 50:45-48, 1993 


\section{Introduction}

4. Deiber MP, Pollak P, Passingham R, et al: Thalamic stimulation and suppression of parkinsonian tremor. Evidence of a cerebellar deactivation using positron emission tomography. Brain 116:267-279, 1993

5. Di Chiro G: Positron emission tomography using $\left[{ }^{18} \mathrm{~F}\right]$ fluorodeoxyglucose in brain tumors. A powerful diagnostic and prognostic tool. Invest Radiol 22:360-371, 1987

6. Di Chiro G, Oldfield E, Wright DC, et al: Cerebral necrosis after radiotherapy and/or intraarterial chemotherapy for brain tumors: PET and neuropathologic studies. AJR 150:189-197, 1988

7. Doyle WK, Budinger TF, Valk PE, et al: Differentiation of cerebral radiation necrosis from tumor recurrence by ${ }^{18} \mathrm{~F}$ FDG and ${ }^{82} \mathrm{Rb}$ positron emission tomography. J Comput Assist Tomogr 11:563-570, 1987

8. Henry TR, Mazziotta JC, Engel J Jr: Interictal metabolic anatomy of mesial temporal lobe epilepsy. Arch Neurol 50: 582-589, 1993

9. Lenzi GL, Frackowiak RSJ: Applications of positron emission tomography to neurosurgery. J Neurosurg Sci 26:237-243, 1982

10. Ogawa T, Uemura K, Shishido F, et al: Changes of cerebral blood flow, and oxygen and glucose metabolism following radiochemotherapy of gliomas: a PET study. J Comput Assist Tomogr 12:290-297, 1988

11. Patronas NJ, Di Chiro G, Kufta C, et al: Prediction of survival in glioma patients by means of positron emission tomography. J Neurosurg 62:816-822, 1986

12. Pirotte B, Goldman S, David P, et al: Stereotactic brain biopsy guided by positron emission tomography (PET) with [F-18]fluorodeoxyglucose and [C-11]methionine. Acta Neurosurg Suppl 68:133-138, 1997

13. Phelps ME, Hoffman EJ, Mullani NA, et al: Application of annihilation coincidence detection to transaxial reconstruction tomography. J Nucl Med 16:210-224, 1975

14. Phelps ME, Mazziotta JC: Positron emission tomography: human brain function and biochemistry. Science 228:799-809, 1985

15. Rasey JS, Koh WJ, Evans ML, et al: Quantifying regional hypoxia in human tumors with positron emission tomography of [18F]fluoromisonidazole: a pretherapy study of 37 patients. Int J Radiat Oncol Biol Phys 36:417-428, 1996

16. Savic I, Ingvar M, Stone-Elander S: Comparison of [11C]flumazenil and [18F]FDG as PET markers of epileptic foci. J Neurol Neurosurg Psychiatry 56:615-621, 1993

17. Sokoloff L, Reivich M, Kennedy C, et al: The [14C] deoxyglucose method for the measurement of local cerebral glucose utilization: theory, procedure, and normal values in the conscious and anesthetized albino rat. J Neurochem 28:897-916, 1977

18. Spence AM, Eary JF, Mankoff DA, et al: 2-[C-11]-Thymidine PET studies of cellular proliferation in gliomas. Neurooncology 1:340, 1999 (Abstract)

19. Stelzer KJ, Tralins K, Mankoff, DA, et al: [F-18]-Fluorodeoxyglucose positron emission tomography (FDG-PET) volume as a predictor of time to progression for glioblastoma multiforme (GM). Neurooncology 1:341, 1999 (Abstract)

20. Ter-Pogossian MM: The origins of positron emission tomography. Semin Nucl Med 22:140-149, 1992

21. Ter-Pogossian MM, Phelps ME, Hoffman EJ: A positron-emission transaxial tomograph for nuclear medicine imaging (PETT). Radiology 114: 89-98, 1975

22. Warburg O: The Metabolism of Tumours. New York: Richard R. Smith, 1931, pp 129-169

23. Xiong J, Nickerson LDH, Downs JH III, et al: Basic principles and neurosurgical applications of positron emission tomography. Neurosurg Clin North Am 8:293-306, 1997 